PROCEEDINGS OF THE

AMERICAN MATHEMATICAL SOCIETY

Volume 127, Number 9, Pages 2557-2560

S 0002-9939(99)05402-7

Article electronically published on May 4, 1999

\title{
A LOWER BOUND FOR THE NUMBER OF COMPONENTS OF THE MODULI SCHEMES OF STABLE RANK 2 VECTOR BUNDLES ON PROJECTIVE 3-FOLDS
}

\author{
E. BALLICO AND R. M. MIRÓ-ROIG \\ (Communicated by Ron Donagi)
}

\begin{abstract}
Fix a smooth projective 3 -fold $X, c_{1}, H \in \operatorname{Pic}(X)$ with $H$ ample, and $d \in \mathbf{Z}$. Assume the existence of integers $a, b$ with $a \neq 0$ such that $a c_{1}$ is numerically equivalent to $b H$. Let $M\left(X, 2, c_{1}, d, H\right)$ be the moduli scheme of $H$-stable rank 2 vector bundles, $E$, on $X$ with $c_{1}(E)=c_{1}$ and $c_{2}(E) \cdot H=$ $d$. Let $m\left(X, 2, c_{1}, d, H\right)$ be the number of its irreducible components. Then $\lim \sup _{d \rightarrow \infty} m\left(X, 2, c_{1}, d, H\right)=+\infty$.
\end{abstract}

The proof that the moduli scheme of rank $r$ stable vector bundles on $S$ with fixed $c_{1}$ and fixed polarization is irreducible for large $c_{2}$ was a major result in the theory of stable vector bundles on an algebraic surface $S$ (see [GL] for the rank 2 case, and $[\mathrm{O}]$ for arbitrary ranks). The result is not true for higher dimensional varieties. In fact there are examples where the minimal number of irreducible components of the moduli scheme of stable vector bundles with some invariants fixed (e.g. $c_{1}$ for the rank 2 case) and the others going to infinity grows to $+\infty$ (see [E] for the rank 2 case on $\mathbf{P}^{3}$ and $[\mathrm{AO}]$ for the rank 3 case on $\mathbf{P}^{5}$ ). It seems to us that this should be the rule and that, for a fixed dimension $n$ and rank $r$, there are less irreducible components of the moduli scheme of rank $r$ stable vector bundles on $\mathbf{P}^{n}$ than on the other $n$-dimensional projective manifolds. Furthermore, there should be more irreducible components of the moduli scheme of stable vector bundles on a fixed manifold if we increase the rank. Here is our result which was strongly inspired by L. Ein's paper [E].

Theorem 0.1. Assume characteristic 0. Fix a smooth projective 3 -fold $X, c_{1}$, $H \in \operatorname{Pic}(X)$ with $H$ ample, and $d \in \mathbf{Z}$. Assume the existence of integers $a, b$ with $a \neq 0$ such that $a c_{1}$ is numerically equivalent to bH. Let $M\left(X, 2, c_{1}, d, H\right)$ (or just $M(d)$ ) be the moduli scheme of $H$-stable rank 2 vector bundles, $E$, on $X$ with $c_{1}(E)=c_{1}$ and $c_{2}(E) \cdot H=d$. Let $m\left(X, 2, c_{1}, d, H\right)$ (or just $m(d)$ ) be the number of its irreducible components. Then $\limsup _{d \rightarrow \infty} m(d)=+\infty$.

We know that Theorem 0.1 is true for $X=\mathbf{P}^{3}$ because L. Ein in [E] constructed enough vector bundles (and called them generalized null correlation bundles). For

Received by the editors October 4, 1997.

1991 Mathematics Subject Classification. Primary 14J60, 14 F05.

Key words and phrases. Vector bundle, stable vector bundle, moduli scheme, $H$-stable vector bundle, projective 3-fold, stability. 
extending Ein's results to arbitrary 3-folds we use that every 3-fold $X$ has a finite map $f: X \rightarrow \mathbf{P}^{3}$. Let $M\left(\mathbf{P}^{3},-1, c_{2}\right)$ be the moduli space of rank 2 stable vector bundles, $E$, on $\mathbf{P}^{3}$ with $c_{1}(E)-1$ and $c_{2}(E)=c_{2}$. Let $\pi: Y \rightarrow \mathbf{P}^{3}$ be the Galois covering of $f: X \rightarrow \mathbf{P}^{3}$. Hence $\pi$ factors through $f$ and we will see in (1.6) how to reduce Theorem 0.1 for $X$ to a corresponding statement for $Y$. The 3 -fold $Y$ may be singular. Take one of the vector bundles, say $B \in M\left(\mathbf{P}^{3},-1, c\right)$, on $\mathbf{P}^{3}$ constructed by Ein. It was proved in [E], Th. 3.2 (b), that the corresponding modular space is smooth at $B$. We will use this result to show that the local deformation space of $\pi^{*}(B)$ is smooth. Using [E], Th. 3.2(b), we will show that if $B^{\prime}$ is another such bundle on $\mathbf{P}^{3}$ with the same Chern classes as $B$ but in a different component of $M\left(\mathbf{P}^{3},-1, c\right)$, then the local deformation spaces of $\pi^{*}(B)$ and $\pi^{*}\left(B^{\prime}\right)$ are smooth and they may be represented by smooth irreducible algebraic varieties, say $U(B)$ and $U\left(B^{\prime}\right)$, parametrizing injectively vector bundles on $Y$ and such that no vector bundle represented by a point of $U(B)$ is isomorphic to a vector bundle represented by a point of $U\left(B^{\prime}\right)$. This would be sufficient to prove the main result on $Y$ and hence on $X$.

The first author was partially supported by MURST and GNSAGA of CNR (Italy). He thanks the Universitat de Barcelona (where this paper was started) and his colleagues there for support and friendly essential help. The second author was partially supported by DGICYT PB91-0231-C02.02. She thanks the Department of Mathematics of the University of Trento for its hospitality during the preparation of part of this work. Both authors want to thank the referee of a previous version of this paper for constructive criticism which stimulated us to find a completely different proof.

1.

We work over an algebraically closed base field $\mathbf{K}$ with $\operatorname{char}(\mathbf{K})=0$. We divide the proof of 0.1 into 6 parts.

(1.1) Note that $M\left(X, 2, c_{1}, d, H\right)$ is a bounded family, hence $m(d)$ is a welldefined integer $\geq 0$.

(1.2) Let $\pi: U \rightarrow V$ be a finite surjective map of projective manifolds, $E$ a rank 2 vector bundle on $V$ and $H \in \operatorname{Pic}(V)$ with $H$ ample. Assume that $\pi$ does not factorize through an unramified morphism of degree $>1$. We claim that if $E$ is $H$ stable, then $\pi^{*}(E)$ is $\pi^{*}(H)$-stable. If $\operatorname{dim}(V)=1$, the claim is [L], Remark 2.3. If $\operatorname{dim}(V)>1$ one can use a restriction theorem to suitable high degree hypersurfaces to reduce to the case $\operatorname{dim}(V)=1$.

(1.3) Note that the notion of $H$-stability depends only on the numerical class of $H$ and it is not changed if we take $H^{\otimes t}$ with $t>0$ instead of $H$. In particular we may assume $H$ 2-divisible. We may twist all the vector bundles in $M(d)$ by a fixed power of $H$. Hence without losing generality we may assume that $H$ is very ample and that $c_{1}=-H$. Note, that since $H$ is allowed to be 2-divisible, up to a twist this is not a restriction on the first Chern class of the stable vector bundles we will construct.

(1.4) Fix a general vector $W \subseteq H^{0}(X, H)$ with $\operatorname{dim}(W)=4$ and let $f: X \rightarrow \mathbf{P}^{3}$ be the associated morphism. Let $\pi: Y \rightarrow \mathbf{P}^{3}$ be the Galois cover of $u$, with associated factorization $v: Y \rightarrow X$. We will check here that if $E, E^{\prime}$ are non-isomorphic rank 2 stable vector bundles on $\mathbf{P}^{3}$ with the same Chern classes, then $\pi^{*}(E)$ and $\pi^{*}\left(E^{\prime}\right)$ are not isomorphic. In particular, this would imply that $f^{*}(E)$ and $f^{*}\left(E^{\prime}\right)$ 
are not isomorphic. By Krull-Remak-Schmidt uniqueness of a decomposition into direct factors, this implies also that for every integer $x>0 \pi^{*}(E)^{\oplus x}$ and $\pi^{*}\left(E^{\prime}\right)^{\oplus x}$ are not isomorphic. By $(1.2) \pi^{*}(E)$ and $\pi^{*}\left(E^{\prime}\right)$ are stable and in particular simple. Hence every isomorphism between $\pi^{*}(E)$ and $\pi^{*}\left(E^{\prime}\right)$ induces a $G$-invariant isomorphism between $\mathbf{P}\left(\pi^{*}(E)\right)$ and $\mathbf{P}\left(\pi^{*}\left(E^{\prime}\right)\right)$. Taking the quotient by the $G$-action we obtain an isomorphism between $\mathbf{P}(E)$ and $\mathbf{P}\left(E^{\prime}\right)$ as $\mathbf{P}^{1}$-bundles over $\mathbf{P}^{3}$. This implies $E \cong E^{\prime}(t)$ for some integer $t$ and hence $E \cong E^{\prime}$ because $c_{1}(E)=c_{1}\left(E^{\prime}\right)$ by assumption. Hence we obtain a contradiction.

(1.5) Here we extract from $[\mathrm{Gr}]$ some well-known results on the equivariant cohomology of sheaves on a projective variety $Y$ on which a finite group, $G$, acts. Let $Y$ be a normal irreducible projective variety on which a finite group $G$ acts. Set $x:=\operatorname{card}(G)$. Let $Z:=X / G$ be the quotient variety and $\pi: Y \rightarrow Z$ the quotient map. We assume $\operatorname{deg}(\pi)=x$. We need only the case $Z=\mathbf{P}^{3}$. If $A, A^{\prime}$ are $G$-sheaves on $X$, in $[\mathrm{Gr}], \mathrm{Ch}$. V and $\mathrm{Ch}$. VI, there is a definition of equivariant cohomology groups $H^{i}(Y ; G, A)$ of $A$ and of equivariant Ext-groups $\operatorname{Ext}^{i}\left(Y ; G, A, A^{\prime}\right)$ of $A$ and $A^{\prime}$. Assume $A \cong \pi^{*}(B)$ with $B$ a vector bundle on $Z$. Note that $G$ acts on $\pi_{*}\left(\boldsymbol{O}_{y}\right)$ and that the factor $\boldsymbol{O}_{Z}$ of $\pi_{*}\left(\boldsymbol{O}_{Y}\right)$ is the $G$-invariant part of $\pi_{*}\left(\boldsymbol{O}_{Y}\right)$; indeed, since we are in characteristic 0 we may apply the averaging operator. Using the averaging operator and the projection formula we obtain that the $G$-invariant part $H^{i}(Y, A)^{G}$ of $H^{i}(Y, A)$ is $H^{i}(Z, B)$. By [Gr], Cor. of Prop. 5.2.3 or Cor. of Th. 5.3.1, we have $H^{i}(Y ; G, A)=H^{i}\left(Z, \pi_{*}(A)^{G}\right)=H^{i}(Z, B)$. By [Gr], first spectral sequence in the statement of Th. 5.6.3, we have $\operatorname{Ext}^{1}(Y ; G, A, A) \cong H^{i}(Z, \operatorname{End}(B))$. We want to check that $\operatorname{Ext}^{1}(Y ; G, A, A)$ is the tangent space to the local deformation space (as $G$-sheaf) of $A$. This may be proven by computing the corresponding functor at the dual numbers $\mathbf{K}[\varepsilon] /\left(\varepsilon^{2}\right)$. This computation may be done as in the nonequivariant case using Cech cocycles; the fact that Cech cohomology may be used in the equivariant setting was proved in [Gr], Th. 5.5.6 and Remark II following it. Set $y:=h^{1}(Z, \operatorname{End}(B))$. In our situation (see 1.6) we will have a flat family, $\mathbf{T}$, of non-isomorphic bundles on $Z$ with $\operatorname{dim}(\mathbf{T})=y$ with $B \in \mathbf{T}$. The pull-backs of these bundles will induce a family, $\mathbf{S}$, of $G$-equivariant mutually non-isomorphic bundles on $Y$ containing $A$ with $\operatorname{dim}(\mathbf{S})=y$. Since $y:=\operatorname{dim}\left(\operatorname{Ext}^{1}(Y ; G, A, A)\right)$ this implies that the functor of $G$-equivariant deformations of $A$ is smooth of dimension $y$.

(1.6) Set $Z:=\mathbf{P}^{3}$. Fix integers $a, b, c$, with $c>b \geq a \geq 0$ and set $t:=$ $c^{2}-a^{2}-b^{2}+c-a-b$. In [E], $\S 3$, Ein constructed a smooth variety $N^{\prime}(a, b, c)$ which is Zariski dense in an irreducible component $N(a, b, c)^{\prime}$ of the moduli space of rank 2 vector bundles on $Z$ with $c_{1}=-1$ and $c_{2}=t$ ([E], Th. 3.2). Now take integers $a^{\prime}$, $b^{\prime}, c^{\prime}$ with $c^{\prime}>b^{\prime} \geq a^{\prime} \geq 0, c^{2}-a^{2}-b^{2}+c-a-b=c^{\prime 2}-a^{\prime 2}-b^{\prime 2}+c^{\prime}-a^{\prime}-b^{\prime}$ and $\left(a^{\prime}, b^{\prime}, c^{\prime}\right) \neq(a, b, c)$. We have $N\left(a^{\prime}, b^{\prime}, c^{\prime}\right)^{\prime} \neq N(a, b, c)^{\prime}$ ([E], Th. 3.2). Using this result and the fact (essentially [E], Lemma 3.5) that the number $n(t)$ of all triples $\left(a^{\prime \prime}, b^{\prime \prime}, c^{\prime \prime}\right)$ with $c^{\prime \prime}>b^{\prime \prime} \geq a^{\prime \prime} \geq 0$ and fixed $t:=c^{\prime \prime 2}-a^{\prime \prime 2}-b^{\prime \prime 2}+c^{\prime \prime}-a^{\prime \prime}-b^{\prime \prime}$ has $\lim \sup _{t \rightarrow+\infty} n(t)=+\infty$, L. Ein proved Theorem 0.1 on $\mathbf{P}^{3}$ for odd first Chern class. Fix $E \in N^{\prime}(a, b, c)$ and $E^{\prime} \in N^{\prime}\left(a^{\prime}, b^{\prime}, c^{\prime}\right)$ with $c^{2}-a^{2}-b^{2}+c-a-b=c^{\prime 2}-a^{\prime 2}-b^{\prime 2}+$ $c^{\prime}-a^{\prime}-b^{\prime}$. By $(1.2) f^{*}(E)$ and $f^{*}\left(E^{\prime}\right)$ are $H$-stable. By Ein's result to prove 0.1 it is sufficient to prove that $E$ and $E^{\prime}$ are in different irreducible components of the moduli space of $H$-stable bundles. In order to obtain a contradiction we assume the existence of a flat family, $\mathbf{U}$, of rank 2 vector bundles on $X$ parametrized by an integral variety $U$ with $f^{*}(E) \in \mathbf{U}$ and $f^{*}\left(E^{\prime}\right) \in \mathbf{U}$. Pulling back this 
family using the morphism $v: Y \rightarrow X$ we obtain a flat family, $\mathbf{W}$, of rank 2 vector bundles on $X$ parametrized by $U$ and with $\pi^{*}(E) \in \mathbf{V}$ and $\pi^{*}\left(E^{\prime}\right) \in \mathbf{V}$. For every vector bundle $F \in \mathbf{V}$, the vector bundle $F[G]:=\bigoplus_{g \in G} g^{*}(F)$ is a $G$-equivariant vector bundle on $Y$. Note that $\pi^{*}(E)[G] \cong \pi^{*}(E)^{\oplus x}$ and $\pi^{*}\left(E^{\prime}\right)[G] \cong \pi^{*}\left(E^{\prime}\right)^{\oplus x}$. Hence we obtain a flat family, $\mathbf{T}$, of vector bundles parametrized by an irreducible variety $U$ and containing both $A:=\pi^{*}(B)$ with $B=E^{\oplus x}$ and $A^{\prime}:=\pi^{*}\left(B^{\prime}\right)$ with $B^{\prime}=E^{\prime \oplus x}$. Take all direct sums of $x$ copies of vector bundles of $N^{\prime}(a, b, c)$ and then do the same with $N^{\prime}\left(a^{\prime}, b^{\prime}, c^{\prime}\right)$. By (1.4) we obtain in this way two irreducible families, say $\mathbf{V}$ and $\mathbf{V}^{\prime}$, with $A \in \mathbf{V}, A^{\prime} \in \mathbf{V}^{\prime}, \operatorname{dim}(\mathbf{V})=x\left(\operatorname{dim}\left(N^{\prime}(a, b, c)\right)\right)$, $\operatorname{dim}\left(\mathbf{V}^{\prime}\right)=x\left(\operatorname{dim}\left(N^{\prime}\left(a^{\prime}, b^{\prime}, c^{\prime}\right)\right)\right)$ and all rank $2 x$ vector bundles in $\mathbf{V} \cup \mathbf{V}^{\prime}$ are mutually non-isomorphic. By (1.5) the local deformation space of $A$ (resp. $A^{\prime}$ ) has Zariski tangent space of dimension $x\left(\operatorname{dim}\left(N^{\prime}(a, b, c)\right)\right)\left(\right.$ resp. $\left.x\left(\operatorname{dim}\left(N^{\prime}\left(a^{\prime}, b^{\prime}, c^{\prime}\right)\right)\right)\right)$. Since these families are algebraic families, this contradicts (1.5) and the existence of the irreducible family $\mathbf{T}$. Hence the proof of 0.1 is complete.

\section{REFERENCES}

[AO] V. Ancona and G. Ottaviani, The Horrocks bundles of rank 3 on $\mathbf{P}^{5}$, J. Reine Angew. Math. 460 (1995), 69-92. MR 96d:14038

[E] L. Ein, Generalized null correlations bundles, Nagoya Math. J. 111 (1988), 13-24. MR 89k:14024

[GL] D. Gieseker and J. Li, Irreducibility of moduli of rank two vector bundles, J. Diff. Geom. 40 (1994), 23-104. MR 95f: 14068

[Gr] A. Grothendieck, Sur quelques points d'algèbre homologique, Tohoku Math. J. 9 (1957), 119-221. MR 21:1328

[L] H. Lange, On stable and ample vector bundles of rank 2 on curves, Math. Ann. 238 (1978), 193-202. MR 80c: 14012

[O] K. O'Grady, Moduli of vector bundles on projective surfaces: some basic results, Invent. Math. 123 (1996), 141-207. MR 96k:14004

Department of Mathematics, University of Trento, 38050 Povo, Trento, Italy

E-mail address: ballico@science.unitn.it

Departamento de Algebra i Geometria, Universitat de Barcelona, Gran Via 585, 008007 BARCELONA, Spain

E-mail address: miro@cerber.ub.es 